\title{
PROPERTIES OF HURWITZ POLYNOMIALS WITH APPLICATION TO
} STABILITY THEORY

\author{
Pankaj kumar $^{1 *}$, Pradeep kumar $^{2}$, Rahul yadav $^{3}$
}

*Corresponding Author: -

\begin{abstract}
: -
In mathematics, a Hurwitz Polynomial, named after Adolf Hurwitz, is a polynomial whose coefficients are positive real numbers and whose roots (zeros) are located in the left half plane of the complex plane or on the jw axis, that is, the real part of every root is zero or negative. Another element of realizability is a class of polynomial known as Hurwitz polynomial which is, in fact, the denominator polynomial of the network function satisfying certain conditions.

1. $P(s)$ is real when $s$ is real.

2. The roots of $p(s)$ have real parts which are zero or negative.

The arguments involve the use of complex plane geometry techniques without invoking the theory of positive paraodd functions or continued fraction expansions methods Some of the established propertiesare then applied to test for the stability of systems of differential equations.
\end{abstract}

\section{(c) $(3)$ (1)}




\section{INTRODUCTION}

Several papers of recent vintage have originated on the eigenvalue clustering and distribution of systems of differential equations The results of are concerned with the extension of the famous Routh array to the complex case The extended Routh array (ERA) was then established and shown to be the naturalcomplex counterpart of the Routh array. The term is sometimes restricted to polynomials whose roots have real parts that are strictly negative, excluding the axis (i.e., a Hurwitz stable polynomial. Hurwitz polynomials are important in control systems theory, because they represent the characteristic equations of stable linear systems. Whether a polynomial is Hurwitz can be determined by solving the equation to find the roots, or from the coefficients without solving the equation by the Routh-Hurwitz stability criterion. The notion of positive para oddness is playing an increasingly effective role in the stability analysis of continuous time systems of differential equations with complex coecients For discrete time systems eigenvalues inside the unit circlethe concept of positive para-oddness is replaced by complex discrete reactancefunctions which are the discrete time counterpart of positive para odd functions

\section{Properties of Network Functions \\ Network Functions:}

A network function is the Laplace transform of an impulse response. Its format is a ratio of two polynomials of the complex frequency s. Consider the general two-port network shown in Figure. The terminal voltages and currents of the two-port can be related by two classes of network functions, namely, the driving point (DP) functions and the transfer functions.

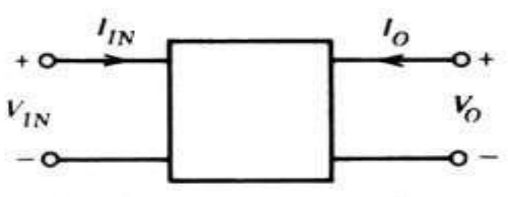

(a) A two port network.

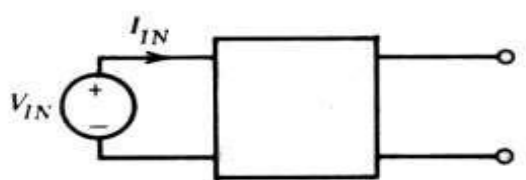

(b) Measuring input impedance.

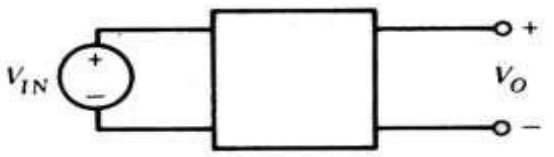

(c) Measuring voltage gain.

Figure 2.2

The DP functions relate the voltage at a port to the current at the same port. Thus, these functions are a property of a single port. For the input port (with the output port an open circuit) the DP impedance function $\mathrm{Z}_{\mathrm{IN}}(\mathrm{s})$ is defined as:

$$
Z_{I N}(s)=\frac{V_{I N}(s)}{I_{I N}(s)}
$$

This function can be measured by observing the current $\mathrm{I}_{\text {IN }}$ when the input port is driven by a voltage source $\mathrm{V}_{\text {IN }}$ (Figure $2.2 b)$. The DP admittance function $\mathrm{Y}_{\mathrm{IN}}(\mathrm{s})$ is the reciprocal of the impedance function, and is given by:

$$
Y_{I N}(s)=\frac{I_{I N}(s)}{V_{I N}(s)}
$$

The transfer functions of the two-port relate the voltage (or current) at one port to the voltage (or current) at the other port. The possible forms of transfer functions are:

1. The voltage transfer function, which is a ratio of one voltage to another voltage.

2. The current transfer function, which is a ratio of one current to another current.

3. The transfer impedance function, which is the ratio of a voltage to a current.

4. The transfer admittance function, which is the ratio of a current to a voltage. The voltage transfer functions are defined with the output port an open circuit,

$$
\begin{gathered}
\text { voltage gain }=\frac{V_{O}(s)}{V_{I N}(s)} \\
\text { voltage loss (attenuation) }=\frac{V_{I N}(s)}{V_{O}(s)}
\end{gathered}
$$

To evaluate the voltage gain, for example, the output voltage $V_{O}$ is measured with the input port driven by a voltage source $\mathrm{V}_{\mathrm{IN}}$ (Figure 2.2c). The other three types of transfer functions can be defined in a similar manner. Of the four types of transfer functions, the voltage transfer function is the one most often specified in the design of filters.

The functions defined above, when realized using resistors, inductors, capacitors, and active devices, can be shown to be the ratios of polynomials in $s$ with real coefficients. This is so because the network functions are obtained by solving simple algebraic node equations, which involve at most the terms $\mathrm{R}, \mathrm{sL}, \mathrm{sC}$ and their reciprocals. The active device, if one exists, the solution still involves only the addition and multiplication of simple terms, which can only lead to a ratio of polynomials in s. In addition, all the coefficients of the numerator and denominator polynomials will be real. Thus, the general form of a network function is: 


$$
\begin{gathered}
H(s)=\frac{a_{n} s^{n}+a_{n-1} s^{n-1}+a_{n-2} s^{n-2}+\cdots+a_{0}}{b_{m} s^{m}+b_{m-1} s^{m-1}+b_{m-2} s^{m-2}+\cdots+b_{0}} \\
\quad \text { where } \quad a_{n} \neq 0 \quad b_{m} \neq 0
\end{gathered}
$$

and all the coefficients $a_{i}$ and $b_{i}$ are real. If the numerator and denominator polynomials are factored, an alternate form of $\mathrm{H}(\mathrm{s})$ is obtained:

$$
H(s)=\frac{a_{n}\left(s-z_{1}\right)\left(s-z_{2}\right) \cdots\left(s-z_{n}\right)}{b_{m}\left(s-p_{1}\right)\left(s-p_{2}\right) \cdots\left(s-p_{m}\right)}
$$

In this expression $\mathrm{z}_{1}, \mathrm{z}_{2}, \ldots, \mathrm{z}_{\mathrm{n}}$ are called the zeros of $\mathrm{H}(\mathrm{s})$, because $\mathrm{H}(\mathrm{s})=0$ when $\mathrm{s}=\mathrm{z}_{\mathrm{i}}$. The roots of the denominator $\mathrm{p}_{\mathrm{l}}$, $\mathrm{p}_{2}, \ldots, \mathrm{p}_{\mathrm{m}}$ are called the poles of $\mathrm{H}(\mathrm{s})$. It can be seen that $\mathrm{H}(\mathrm{s})=\infty$ at the poles, $\mathrm{s}=\mathrm{pi}$.

The poles and zeros can be plotted on the complex s plane $(s=\sigma+j \omega)$, which has the real part $\sigma$ for the abscissa, and the imaginary part $\mathrm{j} \omega$ for the ordinate.

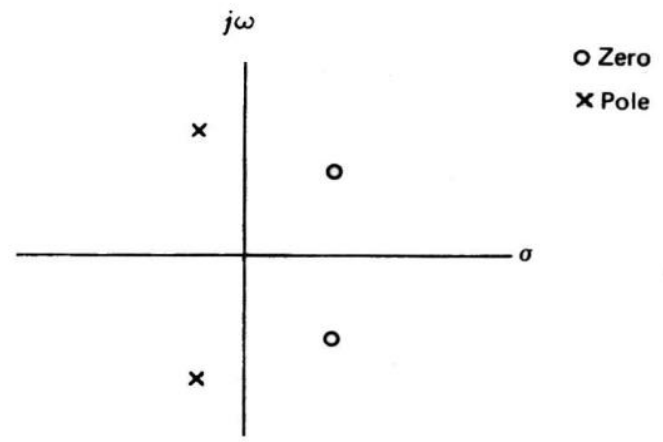

Figure 2.3 Poles and zeros plotted in the complex $s$ plane.

\section{Hurwitz Polynomial:}

A polynomial $\boldsymbol{p}(\boldsymbol{s})$ is said to be Hurwitz if all the roots of $\boldsymbol{p}(\boldsymbol{s})$ are located in the open left half (LH) s-plane (not including the imaginary axis).

$\boldsymbol{p}(\boldsymbol{s})$ is said to be modified Hurwitz if none of its roots are in the open RH s-plane and all imaginary axis roots are simplewith multiplicity equal to one.

In mathematics, a Hurwitz polynomial, named after Adolf Hurwitz , is a polynomial whose coefficients are positive real numbers s and whose roots (zeros) are located in the left half-plane of the complex plane or on the $j \omega$ axis, that is, the real part of every root is zero or negative. The term is sometimes restricted to polynomials whose roots have real parts that are strictly negative, excluding the axis (i.e., a Hurwitz stable polynomial). A polynomial function $P(s)$ of a complex variable $s$ is said to be Hurwitz if the following conditions are satisfied:

1. $P(s)$ is real when $s$ is real.

2. The roots of $P(s)$ have real parts which are zero or negative.

Hurwitz polynomials are important in control systems theory, because they represent the characteristic equations of stable linear systems. Whether a polynomial is Hurwitz can be determined by solving the equation to find the roots, or from the coefficients without solving the equation by the Routh-Hurwitz stability criterion.

A simple example of a Hurwitz polynomial is the following:

The only real solution is -1 , as it factors to

$$
x^{2}+2 x+1
$$

$$
(x+1)^{2} \text {. }
$$

\section{Properties}

For a polynomial to be Hurwitz, it is necessary but not sufficient that all of its coefficients be positive. A necessary and sufficient condition that a polynomial is Hurwitz is that it passes the Routh-Hurwitz stability criterion. A given polynomial can be efficiently tested to be Hurwitz or not by using the Routh continued fraction expansion technique.

1. The odd and even parts of a Hurwitz polynomial $p(s)$ have roots on the jw axis only.if we denote the even part of $\mathrm{p}(\mathrm{s})$ as $\mathrm{M}(\mathrm{s})$ and the odd part as $\mathrm{N}(\mathrm{s})$,

$$
\mathrm{P}(\mathrm{S})=\mathrm{M}(\mathrm{S})+\mathrm{N}(\mathrm{S})
$$

2. Using $\mathrm{M}(\mathrm{s})$ and $\mathrm{N}(\mathrm{s})$ we form the test ratio $\mathrm{T}(\mathrm{s})$, whose numerator has a higher degree than that of its denominator. Suppose that $\mathrm{p}(\mathrm{s})$ is a polynomial of degree $d$. then 


$$
\begin{aligned}
& T(s)=\frac{N(s)}{M(s)} \quad \text { if } d \text { is an odd integer } \\
& T(s)=\frac{M(s)}{N(s)} \quad \text { if } d \text { is an even integer }
\end{aligned}
$$

3. All the poles and zeros are in the left half plane or on its boundary, the imaginary axis.

4. The continued fraction expansion of ratio $T(S)$ of odd to even parts.N(S)/M(S) or the even to odds $M(S) / N(S)$ of a Hurwitz polynomial yields all positive quotient terms.

Network Functions and Their Realizability where qis is the ith quotient, and qi, is the associated coefficient

$$
T(s)=q_{1} s+\frac{1}{q_{2} s+\frac{1}{q_{3} s+\frac{1}{\cdot}}}
$$

5. If there is one or more quotients with negative coefficients, then $\mathrm{p}(\mathrm{s})$ is neither a Hurwitz nor a modified Hurwitz polynomial.

On the other hand, if there are d quotients $\left(d=d^{\wedge}\right)$ and every quotient has a positive coefficient, then $p(s)$ is a Hurwitz polynomial.

Finally, if the number of quotient $d \wedge$ is less than $d$ but every quotient has a positive coefficient, this means that there is a common factor $\mathrm{k}(\mathrm{s})$ between $\mathrm{M}(\mathrm{s})$ and $\mathrm{N}(\mathrm{s})$. Hence, we can write $\mathrm{p}(\mathrm{s})$ as

$$
\begin{gathered}
p(s)=k(s)[\hat{M}(s)+\hat{N}(s)]=k(s) \hat{p}(s) \\
\text { where } M(s)=k(s) \hat{M}(s), N(s)=k(s) \hat{N}(s) \text {, and } \hat{p}(s)=\hat{M}(s)+\hat{N}(s) \text {. }
\end{gathered}
$$

Because all the $\mathrm{d}$ quotients of $\mathrm{T}(\mathrm{s})$ have positive coefficients, the polyno-mial $\mathrm{p}(\mathrm{s})$ in (4-10) is Hurwitz. Thus, if k(s) is a modified Hurwitz polynomial [i.e., if all the roots of $\mathrm{k}(\mathrm{s})$ are simple and purely imaginary], then $\mathrm{p}(\mathrm{s})$ is a modified Hurwitz polynomial.

\section{References}

[1].E. A Guillemin, The Mathematics of Circuit Analysis, $7^{\text {th }}$ edition, Oxford \& IBH Publishing, 1962.

[2].G. A. Korn and T. M. Korn, Mathematical Handbook for Scientists and Engineers, McGraw,New York,1961 .

[3].N. Levinson and R. M. Redheffer, Complex Variable, McGraw Hill Publishing Company Limited,1980.

[4].M. Marden, Geometry of Polynomials, Amer. Math. Soc,2nd edition,1996. [5]. Z Zahreddine. Explicit relationships between Routh Hurwitz and Schour,Cohn types of sta.

[5].Bility, Irish Math. Soc. Bull. 29(1992),49 54

[6].Z. Zahreddine, An extension of the Routh array for the asymptotic stability of a system of differential equations with complex coeffcients, Applicable Analysis, 49(1993),61.72. 